\title{
Quantitative structural analysis of collagen in chordae tendineae and its relation to floppy mitral valves and proteoglycan infiltration
}

\author{
PETER WHITTAKER, ^ DEREK R BOUGHNER, †^ D GARTH PERKINS, \\ PETER B CANHAM* \\ From the Departments of ${ }^{\star}$ Biophysics, $\nmid$ Medicine, and $\ddagger$ Pathology, University of Western Ontario, London, \\ Ontario, Canada
}

SUMMARY Imbibition analysis, a polarised light microscopy technique, was used to examine the molecular organisation of collagen in normal and diseased mitral valve chordae tendineae. $A<$ single strut chorda from each of 23 valves (14 from necropsy specimens and nine from valve replacement surgery) was studied. The degree of molecular organisation of collagen in unstained $7 \mu \mathrm{m}$ sections of the chordae was assessed by measuring the retardation of polarised light by the sample. Sections from each tendon were examined, after staining with Movat's pentachrome, for the presence of proteoglycan infiltration and classified as normal or abnormal on that basis. Theo imbibition analysis results were grouped accordingly. The retardation in the collagen in the sevens chordae with proteoglycan infiltration was significantly lower than in the 16 normal chordae indicating decreased molecular organisation. Five of the seven abnormal chordae with pro-市 teoglycan infiltration and decreased retardation were from patients with floppy mitral valves; the other two were from normal necropsy specimens.

Although proteoglycan infiltration may not be a specific marker for floppy valve disease, its presence is associated with decreased molecular organisation of collagen in the chordae? Degradation of the ground substance bound to the collagen is the most plausible explanation for the measured optical changes.

The widespread occurrence of floppy mitral valves has provoked much interest, both in its clinical aspects and in the pathological process(es) that cause it. No single defect, however, has been identified as the main factor causing floppy valves. Since Read et al first observed mitral prolapse and introduced the term floppy valve, ${ }^{1}$ there have been many suggestions regarding the nature of the abnormality. One histological feature that is frequently associated with floppy valves is the presence of large aggregated amounts of proteoglycans (often called myxomatous infiltration). The accumulation of proteoglycans was first thought to be the primary lesion in floppy

Requests for reprints to Dr Peter Whittaker, Wayne State University Medical School, Myocardial Ischemia Research Laboratory, Cardiology Division, Harper Hospital, 3990 John R, Detroit, MI 48201, USA.

Accepted for publication 20 November 1986 valves. However, myxomatous change is not seen in every floppy valve and may also be found in valves without clinical manifestation of the condition. ${ }^{2}$ 의 Based on their morphological and histological study $\rightarrow$ of mitral valves, King et al proposed that collagen dissolution in the valves and chordae tendineae was $N$ the basic pathological process. ${ }^{2}$ They did not, however, identify the exact nature of the collagen dissolution. Genetic collagen abnormalities such as $\omega$ Marfan syndrome ${ }^{3}$ and the various types of EhlersDanlos syndrome ${ }^{4}$ have also been associated with ${ }^{\circ}$ floppy valves, but the nature of this relation remains $\stackrel{D}{\mathscr{D}}$ unclear. Hammer et al suggested that the collagen in the valve apparatus was abnormal because of an absence of type III and type $\mathrm{V}$ collagen, ${ }^{5}$ but this $\underset{\mathbb{D}}{\mathrm{d}}$ conclusion was based on the study of a single mitral $\stackrel{\circ}{\mathbb{D}}$ valve with ruptured chordae. A later study by Lee $\propto$ et al, with a larger sample size, showed no difference? in the collagen type composition of normal and 8 
floppy valves. ${ }^{6}$ Henney et al found that there was an increased synthesis of collagen in the valve complex. ${ }^{7}$ Others, however, have reported evidence for a breakdown in the normal collagenous structure of the chordae. ${ }^{89}$ These groups used electron microscopy to demonstrate extensive degradation of the collagen in ruptured chordae, which they suggested could be due to enzyme activity. Guthrie and Edwards proposed that chordal elongation observed in mitral valve prolapse was the result of abnormal chordal tension caused by the prolapsing leaflet. ${ }^{10}$ This seems unlikely unless the collagen in the chordae was also abnormal, since mechanical testing has shown that normal chordae are relatively stiff at strain values above $15 \% .^{11}$

The one factor common to these proposals is that collagen structure is a key element in floppy valves. Since the above studies did not reveal a consistent abnormality of collagen fibre structure the problem could be at the molecular level. We used imbibition analysis, a polarised light microscopy technique, to examine the molecular organisation of collagen in the chordae tendineae of normal and floppy mitral valves. This method exploits the anisotropic molecular organisation of collagen, which makes it birefringent and hence visible in the polarising microscope. Changes in molecular organisation that would not be seen with ordinary light microscopy can be observed and quantified by the use of polarised light.

\section{Patients and methods}

A single randomly selected strut chorda was removed from each of 23 mitral valves. Of these specimens, 14 (eight from men and six from women) were obtained at necropsy from patients who had died from non-cardiac causes (age range 26-92), and nine (five men, four women) came from patients undergoing valve replacement for mitral valve disease (age range 58-81). In the latter group, the clinical diagnosis was floppy valve disease in six patients and rheumatic mitral valve disease in the remaining three.

Each of the selected chordae tendineae was fixed in $10 \%$ neutral buffered formalin. The chordae were processed for paraffin embedding and then sectioned longitudinally at a thickness of $7 \mu \mathrm{m}$. Longitudinal sectioning resulted in the collagen fibres lying in the plane of the section. The sections were mounted on strain free glass slides. Immediately before mounting the slides were smeared with albumin fixative, which helped the section adhere to the slide during the measuring procedures. Sections from each of the chordae were then examined by the following techniques.
IMBIBITION ANALYSIS

Theory

When linearly polarised light enters a material such as collagen, which has an anisotropic molecular organisation, the light is resolved into two rays (called the ordinary and extraordinary rays) that are polarised in mutually perpendicular planes. ${ }^{12}$ This property of birefringent materials forms the basis of the method of examining molecular organisation. The two rays travel at unequal velocities, because of the anisotropy of the tissue, and a phase difference is therefore introduced between them. The difference in the two velocities depends on the molecular organisation of the material. Hence, by measuring the retardation produced, which is proportional to the phase difference, the molecular organisation of different samples may be compared. The retardation, $\Gamma$, is given by the formula, $\Gamma=\mathrm{t}\left(\mathrm{n}_{\mathrm{e}}-\right.$ $\left.n_{0}\right)_{i}$ (equation 1 ), ${ }^{12}$ where $t$ is the section thickness and $n_{e}$ and $n_{0}$ are the refractive indices of the two rays. The term $\left(n_{e}-n_{0}\right)_{i}$ is called the intrinsic birefringence and is a characteristic property of the tissue. The measured retardation may be altered by staining and is also dependent on orientation. Therefore, to ensure that the retardation was solely due to the intrinsic molecular organisation of the collagen itself, we studied unstained sections. To eliminate orientation effects the chordae were sectioned longitudinally so that the collagen fibres lay in the section plane.

Form birefringence also contributes to retardation. Form birefringence arises from the difference in refractive index between the material being studied and the surrounding medium. The contribution of form birefringence $\left(n_{e}-n_{0}\right)_{f}$ to the retardation is given by $\left(n_{1}{ }^{2}-n_{2}{ }^{2}\right)^{2} F$ (equation 2 ), where $n_{1}$ is the refractive index of the material, $n_{2}$ the refractive index of the mounting medium, and $F$ is a form factor, ${ }^{13}$ which depends on volume fractions of the different components and the shape of the fibres. The mathematics developed to calculate $\mathbf{F}$ assume that a two component system is being studied-that is the surrounding or imbibing medium and the material. Because of its bound ground substance collagen is a more complex system and the equations cannot be rigorously applied. However, when $n_{1}=n_{2}$ the form birefringence will be zero regardless of the value of $F$, and so only the intrinsic birefringence will contribute to the retardation. Therefore, if retardation in collagen is measured in a series of different media with different refractive indices the retardation at the minimum value on the graph in which retardation is plotted against refractive index will be proportional to the intrinsic birefringence, and hence will be related to the molecular organisation. This procedure is called 
Table The media used in the imbibition analysis and their refractive indices

\begin{tabular}{ll}
\hline Imbibing medium & Refractive index \\
\hline Water & $1 \cdot 33$ \\
$20 \%$ glycerol & 1.36 \\
$40 \%$ glycerol & 1.39 \\
$60 \%$ glycerol & 1.42 \\
$80 \%$ glycerol & 1.44 \\
$100 \%$ glycerol & 1.46 \\
Paraffin oil & 1.48 \\
\hline
\end{tabular}

imbibition analysis and graphs of retardation versus refractive index are called form birefringence curves.

\section{Measuring procedure}

Retardations were measured by the method of de Sénarmont. ${ }^{12}$ A Nikon Optiphot-pol microscope with a $40 \times$ objective, a quarter wave plate, and a monochromatic light source $(\lambda=546 \mathrm{~nm})$ were used. The sections were first cleared of embedding wax with xylene, then washed and soaked in water until the tissue was fully imbibed-that is further soaking produced no change in retardation. The time required to achieve this state was one hour. ${ }^{14}$ The slide was then removed from the water and a coverslip was placed over the section to prevent it from drying. For each section, the retardation of the collagen was measured at ten different locations in the tendon and an average was calculated. The coverslip was then removed, the section was imbibed in another fluid with a higher refractive index, and the retardation measured as before. This process was repeated through a series of imbibing media with successively higher refractive indices. The media used are listed in the table with their refractive indices (measured by an Abbe refractometer). We were guided to our choice of imbibing fluids by the work of Vidal. ${ }^{15}$ Form birefringence curves were drawn for each of the samples. The results from the imbibition analysis were compared by means of $t$ tests.

\section{Picrosirius red staining}

Sections from each of the 23 chordae were stained with picrosirius red, ${ }^{16}$ which is specific for collagen when used with polarised light. ${ }^{17}$ In $7 \mu \mathrm{m}$ sections, normal type I collagen fibres appear orange or red, while thinner degraded collagen fibres will appear yellow or green. ${ }^{18}$ This stain has previously been used to demonstrate collagen degradation by bacterial collagenase in sections of cirrhotic liver ${ }^{19}$ and by leucocytes in the cervix during parturition. ${ }^{20}$ The sections of chordae tendineae were examined for the presence of yellow or green fibres.
Pathological classification

Sections of the 23 chordae were stained with hae $c$ matoxylin and eosin and with Movat's pentachrome $\overrightarrow{\vec{f}_{\text {f }}}$ stain, which contains alcian blue (pH 2.9)-a stain used to demonstrate the presence of proteoglycans $\frac{-}{5}$ Each of the haematoxylin and eosin and Movat' pentachrome stained sections were examined with out knowledge of their source by one of us (DGPO for evidence of proteoglycan infiltration. Each of the chordae was then classified as being normal $0 \vec{B}$ abnormal on the basis of the presence of proteoglycan infiltration.

\section{Results}

The results from the imbibition analysis werf divided into "normal" and "abnormal" group\$ according to the histological classification. The avere age form birefringence curve for each group was then calculated (fig 1). The retardation values for the normal group were significantly higher than fop those that showed proteoglycan infiltration ( $p \&$ $0.05)$. For both curves the minimum retardation $\overrightarrow{0}$ occurred in the pure glycerol solution, correspond ing to a refractive index of 1.46 , which agrees with the findings of others on tendon collagen ${ }^{152122}$ and also with findings from studies of myocardial scar

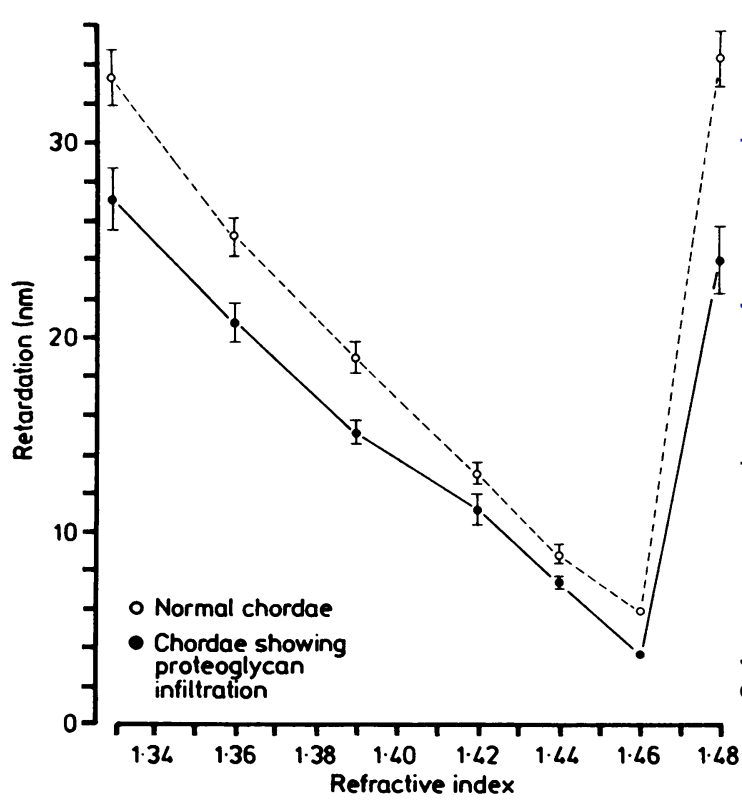

Fig 1 Combined form birefringence curves for collagen sections from normal chordae tendineae and those with proteoglycan infiltration. Where it is large enough the standard error of the mean is shown. 


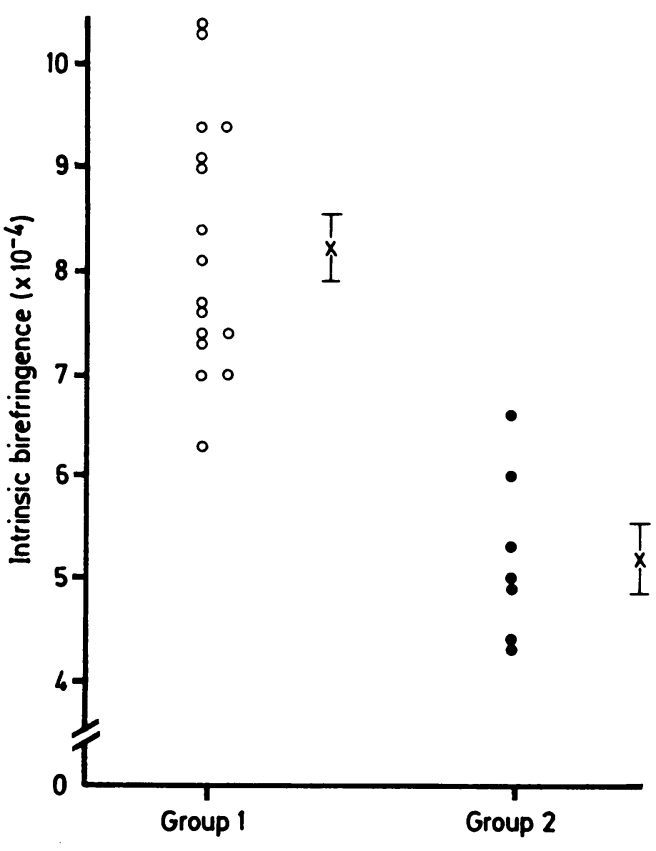

Fig 2 The intrinsic birefringence of the collagen from the normal chordae (group 1) and those showing proteoglycan infiltration (group 2). Mean (SE) is shown for both groups. The intrinsic birefringence was significantly higher $(p<0.001)$ in the normal tendons.

and arterial collagen. ${ }^{14}$ We used the minimum retardation to calculate (equation 1) the intrisic birefringence of the collagen in each of the chordae (fig 2). The average intrinsic birefringence of the abnormal chordae was significantly lower ( $p<$ 0.001 ) than that of the normal chordae. Two chordae showed regions of collagen degradation when stained with picrosirius red-that is some of the fibres appeared green when viewed with polarised light. These regions had been deliberately avoided in the imbibition analysis measurements. Both of these chordae also had proteoglycan infiltration.

Figure 3 shows the correlation of the histological findings with the necropsy and surgical reports. Of the 16 chordae identified as having no evidence of proteoglycan infiltration, five were obtained at the time of replacement surgery and the remaining 11 were from necropsy samples. The surgical reports and the gross pathological descriptions of the surgical specimens from this group showed that three were from stenotic mitral valves and two were from valves with features compatible with floppy valves.

Of the seven chordae that showed proteoglycan infiltration, four were from surgical specimens all of which had been identified as floppy valves and the other three were from necropsy specimens. The pathological report on one of the latter described the specimen as being a floppy valve.

In summary, collagen in all of the sections with proteoglycan infiltration had lower intrinsic birefringence values than sections with no proteoglycan infiltration. This indicates that at the molecular level collagen in such sections is less organised than normal.

\section{Discussion}

We chose chordae tendineae rather than the mitral valve leaflets themselves for this study because collagen fibres in the chordae are highly aligned. In valve leaflets collagen fibre organisation is multidirectional. Since retardation is dependent on orientation it is difficult to ensure that the true retardation is measured when the fibres do not lie in the section plane.

Involvement of mitral chordae in the myxomatous process is known to be variable, and the finding of some normal chordae in patients with floppy valves is to be expected, especially since we were only able to obtain a single tendon from each valve. Also, localised myxomatous change has been found in otherwise unremarkable valves. ${ }^{223}$

The molecular organisation of the collagen in the chordae tendineae will influence their mechanical properties. The imbibition analysis technique was used to examine that molecular organisation. It is a well established technique in ultrastructure research ${ }^{24}$ and in particular has been used to study the changes occurring in the molecular organisation of collagen in the healing and ageing of rat Achilles tendon. ${ }^{2125}$ The significantly lower intrinsic birefringence values of collagen in the chordae that showed proteoglycan infiltration indicated less molecular organisation in these chordae. There are

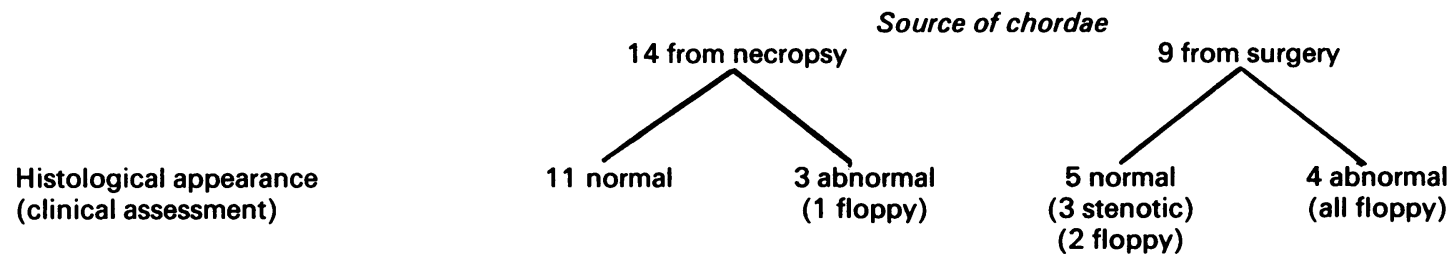

Fig 3 Correlation of the histological findings on the chordae tendineae with the necropsy and surgical reports. 
several possible explanations for this. Firstly, the collagen in the abnormal chordae could be immature. Mello et al have shown that immature collagen has a lower intrinsic birefringence that normal collagen. ${ }^{25}$ Thus our results could be interpreted to mean that the abnormal chordae were composed entirely of immature collagen. This seems unlikely because Doillon et al have shown that around 90 days of age maturing collagen attains retardation values comparable to those of mature collagen. ${ }^{26}$ This would mean that the abnormal chordae were composed of collagen less than three months old. It is difficult to imagine how this situation could occur. The finding of Cole $e t$ al of increased production of collagen and glycosaminoglycans, plus cell proliferation in the valve,$^{27}$ does, however, suggest that a repair process is occurring. A repair process might be associated with the presence of immature collagen and this could be partially responsible for the lower intrinsic birefringences observed. Immature collagen is more extensible and weaker than mature collagen ${ }^{26}{ }^{28}$; therefore, its presence might enable the valve leaflets to prolapse into the atrium.

The second possible explanation is that the altered intrinsic birefringence was due to collagen degradation. This would also lead to increased extensibility and a decrease in the tensile strength. In vivo such a change could result in increased chordal strain at any given stress, causing the chordae to lengthen and the valve cusps to prolapse into the atrium. With extensive collagen degradation, chordal rupture could occur. Obvious collagen degradation was seen in only two of the abnormal samples when they were stained with picrosirius red, but since this method is dependent on collagen fibre diameter ${ }^{18}$ it may not be able to detect the early stages of degradation.

The third possible explanation, and the one that appears most plausible, is that the initial degradation does not involve the collagen per se but rather represents the removal of ground substance bound to the collagen fibre. It has been shown that proteoglycans are bound by collagen fibres in an organised manner, which contributes to the birefringence. ${ }^{1522}$ Therefore, if the ground substance is removed from the collagen the intrinsic birefringence would decrease. The proteoglycan "pools" characteristic of myxomatous infiltration could be regions in which the removed proteoglycans accumulate. Removal of ground substance bound to the collagen could also explain the difference in the shape of the form birefringence curves. The slope of the curve for the normal chordae was steeper than that for the abnormal chordae. Although further experiments would be required to determine the importance of these changes, the shape of the curve for the abnormal chordae is similar to those reported by Vidal, who used enzymes to remove bound ground substanct from normal rat Achilles tendons. ${ }^{22}$ Ground sub stance is known to play an important role in the mechanical properties of tendons. ${ }^{29}$ Treatment 0 human Achilles tendon with $\alpha$ amylase (which removes structural glycoproteins from collagen) has been found to cause a substantial reduction (up t $\$$. $38 \%$ ) in breaking strength and an increase in exten $\vec{\odot}$ sibility. ${ }^{30}$ In chordae tendineae, this effect would allow the chordae to extend and the valve cusps te prolapse.

In summary, our results indicate that, althoug proteoglycan infiltration may not be a specific marker for floppy valves, its presence is associated with evidence of degradation of the collagenous matrix. This finding agrees with the opinion of King $e t a l^{2}$ and suggests that the extent of valvar. involvement in this process is likely to be a major factor in determining the functional effect. It is yêم to be determined whether it is the collagen itself that is degraded or whether it is the ground substance that is removed from the collagen. The polarised light technique of imbibition analysis provided datø not obtainable by the scanning electron microscop studies that have been used to investigate the ultra structure of floppy mitral valves.

This work was supported by a grant from the Hear and Stroke Foundation of Ontario. DRB is ? research associate of that foundation. We thank $D$ F N McKenzie, Dr G Guiraudon, and Miss Elaing Yamamoto for supplying the surgical specimens.

\section{References}

1 Read RI, Thal AP, Wendt VE. Symptomatic valvulă myxomatous transformation (the floppy valve syndrome). A possible forme fruste of the Marfan syndrome. Circulation 1965;32:897-910.

2 King BD, Clark MA, Baba N, Kilman JW, Wooley CF "Myxomatous" mitral valves: collagen dissolution the primary defect. Circulation 1982;66:288-96.

3 Simpson JW, Nora JJ, McNamara DG. Marfan's syñ drome and mitral valve disease: acute surgicab emergencies. Am Heart $\mathcal{F}$ 1969;77:96-9.

4 Leier CV, Call TD, Fulkerson PK, Wooley CF. The spectrum of cardiac defects in the Ehlers-Danlos syndrome, Types I and III. Ann Intern Me 1980;92:171-8.

5 Hammer D, Leier CV, Baba N, Vasko CF, Wooley CH, Pinnell SR. Altered collagen composition in a prot lapsing mitral valve with ruptured chordae ten dineae. Am $\mathcal{f}$ Med 1979;67:863-6.

6 Lee YS, Lee AH, Lu AH, et al. Biochemical analys and electron microscopy of human mitral valve co $\mathrm{P}$ lagen in patients with various etiologies of mitrag valve disease. $\mathcal{F p n}$ Heart $\mathcal{F}$ 1983;24:529-38. 
7 Henney AM, Parker DJ, Davies MJ. Collagen biosynthesis in normal and abnormal human heart valves. Cardiovasc Res 1982;16:624-30.

8 Caulfield JB, Page DL, Kastor JA, Sanders CA. Connective tissue abnormalities in spontaneous rupture of chordae tendineae. Arch Pathol 1971;91:537-41.

9 Scott-Jupp W, Barnett NL, Gallagher PJ, Munro JL, Ross JL. Ultrastructural changes in spontaneous rupture of mitral valve chordae tendineae. $\mathcal{f}$ Pathol 1981;133:185-201.

10 Guthrie RB, Edwards JE. Pathology of the myxomatous mitral valve. Minn Med 1976;59:637-47.

$11 \mathrm{Lim} \mathrm{KO}$, Boughner DR. Mechanical properties of human mitral valve chordae tendineae: variation with size and strain rate. Can $\mathcal{f}$ Physiol Pharmacol 1975;53:330-9.

12 Bennett HS. The microscopical investigation of biological materials with polarized light. In: McClungJones R, ed. McClung's handbook of microscopical technique. New York: PB Hoeber, 1950:591-677.

13 Bendet IJ, Bearden I. Birefringence of spermatozoa. f Cell Biol 1972;55:501-10.

14 Whittaker P. Structural analysis of cardiovascular tissue using quantitative polarised light microscopy. University of Western Ontario, 1986. Ph.D. Thesis.

15 Vidal BC. The part played by proteoglycans and structural glycoproteins in the macromolecular orientation of collagen bundles. Cell Mol Biol 1980;26: 415-21.

16 Sweat F, Puchtler H, Rosenthal SI. Sirius red F3BA as a stain for connective tissue. Arch Pathol 1964;78: 69-72.

17 Junqueira LCU, Bignolas G, Bretani R. Picrosirius staining plus polarizing microscopy, a specific method for collagen detection in tissue sections. Histochem $\mathcal{F}$ 1979;11:447-55.

18 Junqueira LCU, Montes GS, Sanchez EM. The influence of tissue section thickness on the study of collagen by the picrosirius-polarization method. Histochemistry 1982;74:153-6.

19 Perez-Tamayo R, Montfort I. The susceptibility of hepatic collagen to homologous collagenase in human and experimental cirrhosis of the liver. Am $\mathcal{f}$ Pathol 1980;100:427-40.
20 Junqueira LCU, Zugaib M, Montes GS, Toledo OMS, Krisztan RM, Shigihara RM. Morphology and histochemical evidence for the occurrence of collagenolysis and for the role of neutrophilic polymorphonuclear leukocytes during cervical dilation. Am f Obstet Gynecol 1980;138:273-81.

21 Mello MLS, Vidal BC, de Carvalho AC, Caseior-Filho AC. Change with age of anisotropic properties of collagen bundles. Gerontology 1979;25:2-8.

22 Vidal BC. The part played by the mucopolysaccharides in the form birefringence of collagen. Protoplasma 1965;59:472-9.

23 Malcolm AD. Mitral valve prolapse associated with other disorders: casual coincidence, common link, or fundamental genetic disturbance? $\mathrm{Br}$ Heart $\mathcal{f}$ 1985;53:353-62.

24 Frey-Wyssling A. Ultrastructure research in biology before the electron microscope. I Microsc 1974;100: 21-34.

25 Mello MLS, Godo C, Vidal BC, Abujadi JM. Changes in the macromolecular orientation of collagen fibres during the process of tendon repair in the rat. Ann Histochem 1975;20:145-52.

26 Doillon CJ, Dunn MG, Bender E, Silver FH. Collagen fiber formation in repair tissue: development of strength and toughness. Collagen Relat Res 1985;5: 481-92.

27 Cole WG, Chan D, Hickey AJ, Wilcken DEL. Collagen composition of normal and myxomatous human mitral heart valves. Biochem $\mathcal{f}$ 1984;219: $451-60$.

28 Gillman T. On some aspects of collagen formation in localized repair and in diffuse fibrotic reactions to injury. In: Gould BS, ed. Treatise on collagen. Vol. 2, part B. New York: Academic Press, 1968:331-407.

29 Viidik A, Danielson CC, Oxlund H. On fundamental and phenomenological models, structure and mechanical properties of collagen, elastin and glycosaminoglycan complexes. Biorheology 1982;19: 437-51.

30 Minns RJ, Soden PD, Jackson DS. The role of fibrous components and groundsubstance in the mechanical properties of biological tissue: preliminary investigation. F Biomech 1973;6:153-65. 